\title{
Training Situation Center BaSEd ON THREe-Dimensional VIRTUAL STUDIO FOR DiSTRIBUTED MOBILE ROBOTICS LABORATORY
}

\author{
Victor Andreev ${ }^{\mathrm{a}, \mathrm{b}, \mathrm{e}}$, Viacheslav Karbanov ${ }^{\mathrm{c}}$, Konstantin Kharin ${ }^{\mathrm{b}}$, Sergey Kuvshinov ${ }^{\mathrm{a}, \mathrm{b}}$, \\ Yury Poduraev ${ }^{\mathrm{a}}$, Valentin Pryanichnikov ${ }^{\mathrm{a}, \mathrm{b}, \mathrm{d}, \mathrm{e}, \mathrm{f}}$ \\ "MSTU "STANKIN", Vadkovsky lane 1, Moscow, 127994, Russia \\ ${ }^{b}$ International Institute of New Educational Technologies, RSUH, Miusskaya sq. 6, Moscow, 125993, Russia \\ ${ }^{c}$ Telemetrics Inc, Camera Control Systems, 6 Leighton Place, Mahwah, New Jersey 07430 \\ ${ }^{d}$ Keldysh Institute of Applied Mathematics, RAS, Miusskaya sq. 4, Moscow, 125047, Russia \\ 'International Laboratory "Sensorika”, Miusskaya sq. 4, Moscow, 125047, Russia \\ ${ }^{f}$ University of Zadar, Mihovila Pavlinovica bb, Zadar, 23000 Croatia
}

\begin{abstract}
We consider the problems of creating a training situation center for training management skills of mobile robotic systems control based on the concept of territorially distributed laboratory. This task can be efficiently performed by means of three-dimensional rendering system and software and hardware support for information analysis and management activities. The base concept of approach is the real-time virtual studio that provides computer simulation of threedimensional scenes and motion of actual mobile robots, real-time operation with multiple cameras and have powerful and fast 3D and stereoscopic real-time rendering. We have developed 3D scenes corresponding to places of the intended use of robotic devices and additionally several polygonal 3D models of external artifacts, textures for them and finally recorded animation layers with appropriate controllers of the virtual studio. Mobile robots moved against a background of the chromakey green screen in different places of distributed laboratory are incorporated into the virtual network laboratory on the basis of VPN-tunnels through the Internet by special software.
\end{abstract}

Keywords: 3D virtual studio; training situation center; mobile robots; territorially distributed laboratory; real-time simulation; local area network
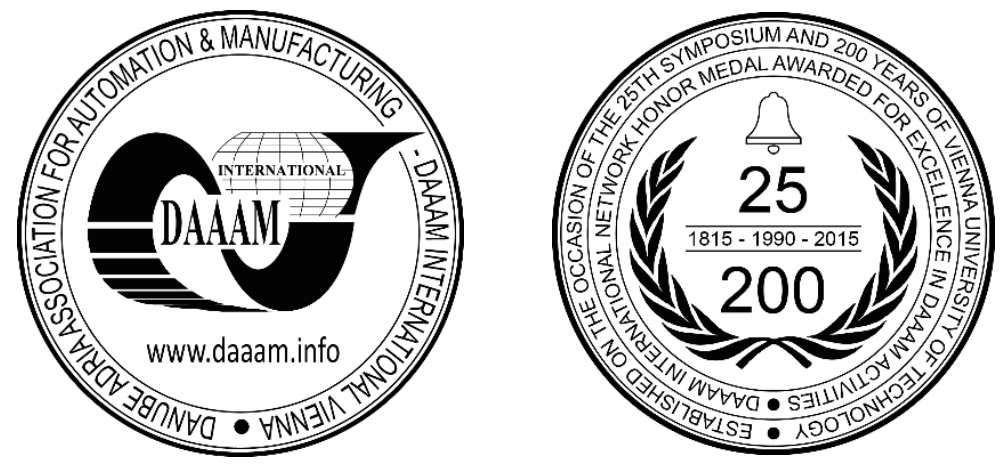

This Publication has to be referred as: Andreev, V[iktor]; Karbanov, V[iacheslav]; Kharin, K[onstantin]; Kuvshinov, S[ergey]; Poduraev, Y[ury] \& Pryanichnikov, V[alentin] (2016). Training Situation Center Based on Three-Dimensional Virtual Studio for Distributed Mobile Robotics Laboratory, Proceedings of the 26th DAAAM International Symposium, pp.0483-0487, B. Katalinic (Ed.), Published by DAAAM International, ISBN 978-3-902734-07-5, ISSN 1726-9679, Vienna, Austria

DOI: $10.2507 / 26$ th.daaam.proceedings.064 


\section{Introduction}

Emergency recovery activities commonly require an operational group of robots. "The investigations conducted by various research centers and companies in the field of robotics have indicated that the creation of a multi-unit group of mobile robots operating as an integrated system is one of the main components in the development of an efficient intelligent unmanned technology capable of operating in combat conditions, including heavy emergencies recovery activities" [1]. Also, in many cases there is a need in immediate help by various experts or access to a specific database. Therefore, the information support of these operations and training of future experts and operators are important tasks. A similar problem is stated in the field of robotic systems designed for space applications. For example, the authors of [2] formulated the main directions of development of robotic systems for space applications, stating particularly that "The solution of technological problems is largely based on ... the development of methods for group control of robotic systems for different space applications as well as their communication; the development of methods for remote control of autonomous robotic systems for space applications on the basis of network technologies, self-diagnostics, and selftraining; the organization of advanced human-machine interfaces ...".

In both cases, the problem can be efficiently solved by creating a training situation center equipped with a threedimensional rendering system and software and hardware support for information analysis and management activities. This formulation of the problem provides a natural basis for its solution using a multi-level hierarchical system for the formation and exchange of information/control data flows. This, in turn, requires a mechanism offering communication between the group of mobile robots and control stations served simultaneously by many operators as well as a situation center as the top of the hierarchy [3]. A prerequisite of this communication is the organization of communication channels that allow the integrated command center of situational control to be located many kilometers away from the operational area. It should be taken into account that the operations are normally performed by mobile robots that should not be restricted in their freedom of motion, which can be ensured by a radio channel. In addition, the capacity of communication channels should ensure that the 3D multistream video can be transmitted from vision systems of mobile robots to both control stations and the situation center.

\section{The structure of the territorially distributed training situation center}

We suppose that this problem can be solved on the basis of network technologies. A key feature of this approach is that all units of the system are mobile nodes of a local area network (LAN). The structure of this center is presented in [4].

For the structure of the situational center, it is quite helpful to use the experience in development and operation of the virtual distributed laboratory at the International Institute of New Educational Technologies in the Russian State University for the Humanities (IINET) and the International Laboratory Sensorika [4, 5]. This virtual laboratory is designed for remote control of mobile robots and training of experts [6,7]. The use of network technologies made it possible to combine multiple mobile robots, accompanying systems ("satellites"), repeaters, and control boards into a local area network. The Internet was taken to be the communication channel. To avoid unauthorized access, the communication was performed through VPN-tunneling. On the basis of VPN-tunneling, an integrated network was deployed consisting of the educational mobile robots located in IINET (Moscow), the Keldysh Institute of Applied Mathematics of the Russian Academy of Sciences (Moscow), and the Far East Federal University (Vladivostok).

The investigations performed on this structure allowed us to formulate the principles for construction and operation of training situation centers for the Federal Atomic Energy Agency (FAEA) and the Ministry of the Russian Federation for Civil Defense, Emergencies and Elimination of Consequences of Natural Disasters (EMERCOM), where different robotic systems are widely used. We developed software allowing not only to remotely observe a threedimensional "scene" but also remotely control these robots by a technology created for overcoming the irregularities and delays in communication channels. The computer network is based on the TCP VIP protocol stack since it is a currently widespread and well-proven solution. The networks that are based on the TCP IIP stack are well scalable and have many technical implementations. It makes possible to combine different hardware and software modules of all units of the system into an integrated network. Then, the problem is reduced to the creation of a local area network with its nodes being mechatronic devices, such as digital IP-cameras, computing devices mounted onboard the robotic device and on remote satellite units, and repeaters, on the one hand, and a computer on the operator console and workstations in the situation center, on the other hand; each with its own IP-addresses belonging to the same network.

\section{Multi-level control system}

Research on the virtual distributed laboratory using three-dimensional rendering technologies has made it possible to create a mechanism of control of multiple mobile robots. For this case, a multilevel control system was constructed [3]. The first level is the mobile robot LAN connecting the mechatronic systems with the informationmeasuring and control system (IMCS) of mobile robots. The second level is the onboard LAN and the LAN of the control station combined through the radio channel. The third level is the LANs of individual robotic systems combined into a single LAN (LAN with mobile nodes) through appropriate communication channels. The fourth level combines the LANs 
of individual groups of mobile robots and the LAN of the situation center into a single LAN via Internet VPN-tunneling. In this structure, the commander can entirely observe a three-dimensional scene and receive video data from vision systems of all mobile robots and satellite modules. The use of LAN on the basis of the TCPIIP protocol stack is convenient since the computer of the commander can be connected to the network at any level and the system components can operate with appropriate network software.

This software was based on the equipment of the training classroom of electronic simulators developed by the International Laboratory Sensorika for the Engineering and Educational Center of FAEA [8]. This classroom is designed for quality and regular training of operators of ground-based robotic systems and remotely controlled systems. In addition to determining the network structure, we arranged a common scene when up to seven robots were controlled by seven operators. The software components interact through the classical mechanism of messaging without the use of parallel execution mechanisms. In this case, the processing of user commands, data transmission over the network etc. are performed not earlier than the current cycle of message processing is completed. Changing the message processing cycle, we were able to significantly reduce the time of response of the robot model to control commands coming from a remote operator $[9,10]$. The structure allows the Internet connection to be organized at any point, i.e., at places where the network is available.

\section{Real-time virtual 3D-studio to practice operator skills}

To practice the skills of mobile robot control under conditions of the training situation center, we use a 3D virtual studio that handles the complex algorithm of real-time motions of mobile robots in the simulated three-dimensional space. Actually, the motion of mobile robots is seen against the background of the chromakey green screen, as shown in Fig. 1.

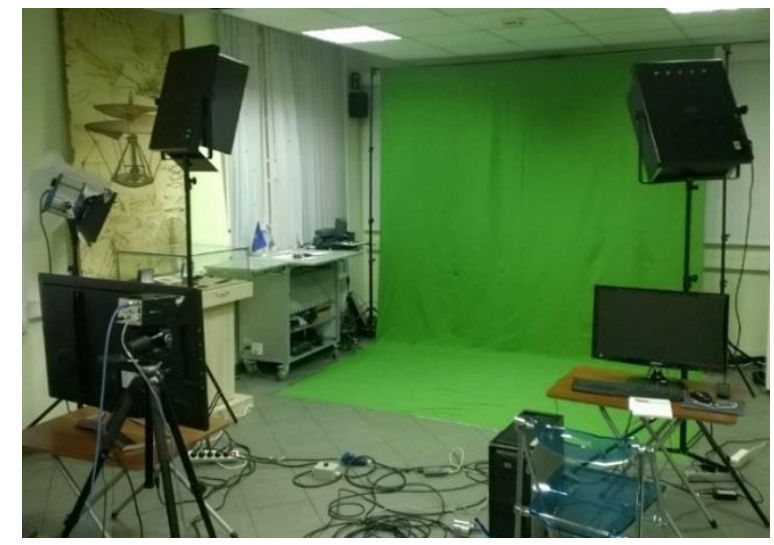

Fig. 1. Equipment of the virtual studio of the training situation center

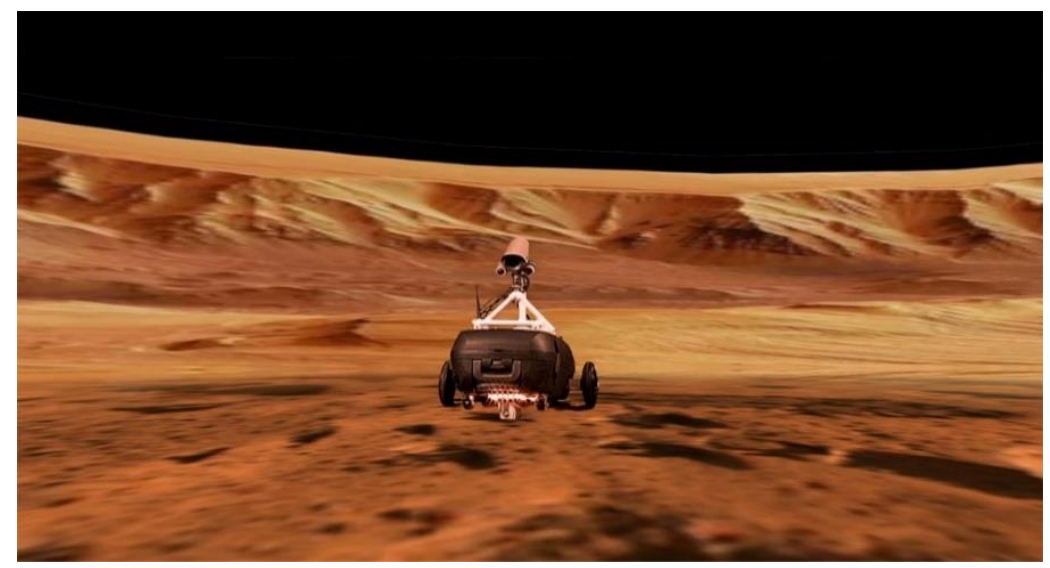

Fig. 2. Model of the training field of the situation center

Images from the external video camera and the camera of the mobile robot are integrated (in the real-time mode and stereo format) into a virtual three-dimensional space, which can be a training field with different terrains and obstacles. An example of a model of the field for practicing the training situation is shown in Fig. 2.

The three-dimensional virtual space of the training situation center became operable through the use of the special-purpose software EasySet3D. It is very important to use the virtual studios and 3D-graphics in real-time simulation systems. The virtual studios are most versatile and adaptable to rendering of 3D scenes and they are subjects of special interest. These virtual studios: 
- provide real-time operation with multiple cameras;

- have powerful and fast 3D and stereoscopic real-time rendering;

- operate both in the interpolation mode and with camera position tracking;

- use an open platform and graphics import programs;

- have a simple and intuitive interface.

EasySet3D fully meets the above-formulated software requirements. The system offers an industry-leading fast 3D and stereo render, uses the HP and Nvidia Quadro open API platform, is equipped with a built-in chromakey, has virtual cameras emulating smooth displacements in a virtual environment, a production mixer with preview, a generator of effects, and many other features of traditional television systems. The user-friendly interface makes it possible to create and import complex and realistic virtual three-dimensional sets just by a few clicks of the computer mouse using expandable libraries of sets and elements that are supplied with the main software. The system combines the simplicity of management, the powerful real-time 3D processing of Brainstorm eStudio, the capability of operating in the economy mode with virtual cameras without using external tracking systems and chromakeying, and the alternative capability of operating in the high-end mode with an external hardware chromakey and camera tracking systems. Also, it should be noted that the virtual cameras of the system are used efficiently and can be moved freely and smoothly from given positions at a desired speed as well as that there is an extensive library of backgrounds, walls, floors, video screens, and ready-to-use virtual sets.

To practice the rendering and mapping into a 3D environment, we have primarily developed 3D scenes corresponding to places of the intended use of robotic devices. In addition to the scene models, we developed several polygonal 3D models of external artifacts; then, we created textures for them and recorded animation layers with appropriate controllers imported into the virtual studio. Also, directly in the virtual studio, we recorded animation effects (so-called productions) in the real-time mode.

At this stage of the creation of the training situation center, no robotized tracking systems were used. This means that the motion of mobile robots was recorded on a green background using a static camera without an operator. Thus, the image on the screen of the mobile device operator remained flat and the effects of "changed shooting perspective" was achieved only by using complex interpolation algorithms that have substantial limitations to their use; i.e., the virtual studio technology was used without tracking. At present, this approach is also accepted as a virtual studio without tracking systems but allowing for a real-time integration of humans and robotic objects placed against a blue or green background into a three-dimensional virtual environment.

EasySet3D affords free movements in the three-dimensional environment with appropriate scaling of objects on the basis of interpolation and special subroutines.

A virtual studio with tracking, or a virtual studio in the classic sense, is a hardware and software system that provides real-time integration of the objects recorded with the real camera motion into a three-dimensional (man-made) computer environment. Here, the resulting video of the virtual camera should be rendered synchronously in real time, in the same perspective; i.e., a change in the shooting angle for a real object leads accordingly to a change in the angle of view of the virtual studio. Thus, at each time, the virtual scene must track the appropriate location of the actual camera along all of its axes of motion and in all modes (pan, tilt, zoom in, zoom out, scaling). Under these conditions, the resulting video will be fully adequate.

Tracking systems can track the position of cameras at each time and have high-frequency sensors detecting the movement of cameras along the three coordinates and all axes of movement. These systems can be constructed on the basis of different principles. Mechanical, optical, magnetic and IR-camera-based (where cameras are located along the virtual studio perimeter and the targets are on the cameras) tracking systems are among most widely used systems. The best results are obtained with tracking systems integrated into special robotized systems with rail-guided, telescopic, and rotary systems.

The level of realism of the virtual studio depends directly on its characteristics and capabilities as well as the graphics and hardware platforms used. Experimental studies indicated that optimal results are achieved with systems that are based on HPz840 workstations with Nvidia Quadro 5000/6000/5K/6K graphics cards. In our opinion, the capture cards of video (HD SDI) and programmed output (SDI Out) are better to be used by Nvidia; in this case, the cards are fully compatible and the speed of combined video processing (video capture, graphical processing, and output) is maximal. Even in terms of economics, when multiple HD SDI video inputs are to be used simultaneously, the Nvidia conveyor is the most appropriate. The practice shows that along with Nvidia, the performance of AJA Kona 3G, BlueFish, and DeckLink cards is also satisfactory [11].

\section{Conclusions}

The technology of real-time 3D virtual studios will allow us to integrate the motion of mobile robots and 3D scene models into a complex computing environment (such as the lunar/Martian landscapes and complex construction terrains) and realistically visualize their interaction.

In the next step, based on the historical experience, the training situation center is supposed to be equipped with a virtual studio with EasySet3D and Infinity software operating on robotized Telemetrics systems (the RCCP1 STS remote camera control system, a range of rotary systems, Teleglide tracking systems, a telescopic system, RoboEye special- 
purpose cameras to work with mobile robots, control interfaces, and base stations with fiber optic communication channels) with built-in tracking options. These systems will provide smooth convergent camera movements with desired speeds and transitions allowing for a full-scale perception of the volume of scenes where the movements of mobile robots occur.

The use of this approach for creating a training situation center will allow the training process to become more efficient and adequate for complex external conditions. This can be achieved by using mobile robotics data processed by distributed local control centers and the system of three-dimensional rendering of the situation and movements of mobile robots in real time and in the stereo mode. This will provide a higher level of training quality for future experts.

\section{References}

[1] Kopylov, N.P. and Tsarichenko, S.G., The conception of robotics progress in the interests of the Ministry of the Russian Federation for Civil Defense, Emergencies and Elimination of Consequences of Natural Disasters, Extreme Robotics. Nano-, Micro-, and Macro-Robots (ER-2009). Proceedings of the XX International Scientific and Technical Conference, Taganrog: TTI YuFU, 2009, pp. 26-28.

[2] Minakov, E.P., Lopota, V.A., Yurevich, E.I., and Kondratev, A.S., The conception of robotics progress in the interests of manned astronautics, exploration of the Moon and planets of the solar system. Extreme Robotics. Nano, Micro-, and Macro-Robots (ER-2009). Proceedings of the XX International Scientific and Technical Conference, Taganrog: TTI YuFU, 2009, pp. 103-106.

[3] Andreev, V.P., Kirsanov, K.B., Pletenev, P.F., Poduraev, Yu.V., Pryanichnikov, V.E., and Prysev, E.A., Technology supervisory control for mechatronic devices via the Internet, 25th DAAAM Int. Symp. on Intelligent Manufacturing and Automation, 2014. Procedia Engineering, vol. 100, 2015, pp. 33-40.

[4] Pryanichnikov, V., Andreev, V., Bobrov, P., Biryukova, E., Frolov, A., Kharin, K., Kirsanov, K., Kostin, A., Kuvshinov, S., Marzanov, Y., and Prysev, E., Scientific-educational distributed laboratory - software and hardware means, Annals of DAAAM for 2012 \& Proceedings of the 23th international DAAAM Symposium "Intelligent Manufacturing \& Automation" 24-27th October 2012 Zadar, Croatia, 2012, vol. 23, no. 1, pp. 11751178.

[5] Vladimir F. Filaretov, Valentin E. Pryanichnikov, Autonomous Mobile University Robots AMUR: Technology and Applications to Extreme Robotics, 25th DAAAM Int. Symp. on Intelligent Manufacturing and Automation, 2014. Procedia Engineering, vol. 100, 2015, pp. 269-277.

[6] Andreev, V., Pryanichnikov, V., Poduraev, Y., and Kuvshinov, S., Education on the basis of virtual learning robotics laboratory and group-controlled robots, 24th DAAAM Int. Symp. on Intelligent Manufacturing and Automation, 2013, Procedia Engineering, 2014, vol. 69, pp. 35-40.

[7] Kuvshinov, S.V, The use of modern IT-technologies for creating an interactive educational environment on the basis of 3D dynamical models, Intelligent and Adaptive Robots, 2009, 1-2, pp. 93-96.

[8] Andreev, V.P., Kirsanov, K.B., Levinsky, B.M., Pryanichnikov, V.E., and Travushkin, A.S., Development of a classroom of electronic simulation training systems on the basis of high-speed LAN, Information-Measuring and Control Systems, 2009, vol. 7, no. 6, pp. 82-92.

[9] Stopper, M. and Katalinic, B., Service-oriented architecture design aspects of OPC UA for industrial applications, Proceedings of the International Multi-Conference of Engineers and Computer Scientists, IMESC-2009, March 1820, 2009, Hong Kong, vol. 2, pp. 1809-1812.

[10] Andreev, V.P., Pryanichnikov. V.E., and Prysev, E.A., Multi-access control of distributed mobile robotic systems based on networking technologies, Annals of DAAAM for 2010 \& Proceedings of the 21st International DAAAM Symposium, Vienna, Austria 2010, Katalinic, B., Ed., Vienna: DAAAM International, 2010, pp. 15-16.

[11] Karbanov, V.A., Virtual studios, 625PlusHD, 2014, no. 1 (195), pp. 18-21. 